\title{
Turning skin into dopamine neurons
}

\author{
Malin Parmar ${ }^{1}$, Johan Jakobsson ${ }^{1}$ \\ ${ }^{1}$ Department of Experimental Medical Science, Wallenberg Neuroscience Center, and Lund Stem Cell Center, Lund University, SE- \\ 22184 Lund, Sweden \\ Cell Research (2011) 21:1386-1387. doi:10.1038/cr.2011.130; published online 9 August 2011
}

The possibility to generate neurons from fibroblasts became a reality with the development of iPS technology a few years ago. By reprogramming somatic cells using transcription factor (TF) overexpression, it is possible to generate pluripotent stem cells that then can be differentiated into any somatic cell type including various subtypes of neurons. This raises the possibility of using donor-matched or even patientspecific cells for cell therapy of neurological disorders such as Parkinson's disease (PD), Huntington's disease and stroke. Supporting this idea, dopamine neurons, which are the cells dying in $\mathrm{PD}$, derived from human iPS cells have been demonstrated to survive transplantation and reverse motor symptoms in animal models of PD [1]. However, a major problem with the use of pluripotent cells as starting material for cell replacement therapy is their incomplete differentiation and their propensity to form tumors following transplantation [2]. In a recent issue of Nature, Caiazzo et al. describe an alternative way to reprogram fibroblasts into dopaminergic neurons, without going through a pluripotent stage [3].

Caiazzo et al. overexpressed three transcription factors, Mash1, Nurr1 and Lmx1a, in fibroblasts and found

Correspondence: Malin Parmar ${ }^{\text {a }}$ Johan Jakobsson $^{\mathrm{b}}$

${ }^{\mathrm{a} E}$-mail: malin.parmar@med.lu.se

bE-mail: johan.jakobsson@med.lu.se efficient and rapid conversion into dopaminergic neurons and show that conversion takes place without going through a pluripotent or even a progenitor cell stage. The induced dopamine neurons displayed several hallmarks of functional dopamine neurons in vitro, including morphology, marker gene expression, electrophysiological and biochemical properties. Importantly, their strategy appeared to be successful in fibroblasts from both mouse and human donors including cells from aged individuals suffering from Parkinson's disease.

The study from Caiazzo et al. is a recent addition to a series of publications that demonstrate that direct conversion of fibroblasts into neurons is feasible [3-6]. In a seminal publication by Vierbuchen et al., three transcription factors were also used, in this case Mash1, Brn2, Myt11, to reprogram mouse fibroblasts into excitatory functional neurons [6]. In a follow up study they then found that the same three factors, with the addition of NeuroD1, were able to generate functional neurons from human fibroblasts [4]. In a recent paper we have used five factors (Mash1, Brn2, Myt11, Lmx1a, FoxA2) to generate dopamine neurons from human fibroblasts [5]. Thus transcription factor mediated conversion of fibroblasts to neurons appears to be a solid phenomenon that is reproducible among several labs. When looked upon together, these studies firmly establish induced neuronal (iN) cells as a way to generate subtype specific neurons from individuals, thereby allowing for disease modeling, drug screens and as a promising candidate approach for cell therapy of neurological disorders.

The initial iN reports clearly demonstrate that cells with functional characteristics of neurons are formed [4-6], but it has up until now remained unclear to what extent the cells are truly reprogrammed into bona fide neurons. The paper by Caiazzo et al. provides the first insight on the extent of reprogramming in iN cells by performing global mRNA-array analysis on the converted cells [3]. They find that while certain dopamine-related genes are induced and some fibroblasts genes are lost, there are still major transcriptional discrepancies between induced dopamine neurons and the native counterparts. The functional consequences of this discrepancy remain unclear, but may affect neuronal properties as well as cell viability and function. In future studies it will be important to assess the degree of reprogramming also in the context of epigenomic and proteomic studies as data from the iPS field suggest that incomplete reprogramming has major impact on the properties of the cells [7]. Functionality and long-term survival of dopaminergic iN cells produced by different transcription factor cocktails are still to be shown and is best analyzed in transplantation experiments. It is promising that Caiazzo et al. provide the first evidence that converted mouse neurons 
can survive transplantation into neonatal brain [3]. It will be interesting to know if human iN cells survive transplantation into a parkinsonian brain and if the grafted cells can revert motor symptoms in animal models of PD.

Still, the mechanisms that drive conversion of fibroblasts into neurons remain unexplored and it is unclear if the optimal combinations of TFs have been used so far. Although the abovementioned studies use different combinations of genes all of them in part rely on Mash1 [3-6]. Overexpression of Mash1 alone in fibroblasts appears to initiate a limited transformation towards immature neuronal cells and the additional factors are then needed to govern maturation and subtype specification [6]. Mash1 is one of several proneural basic helix-loop-helix transcription factors that functions in specifying neuronal identity. Interestingly, Mash1 is expressed in spatially restricted domains in the developing brain while other proneural genes, such as Ngn2, fill the same function in areas where Mash1 is not expressed [8]. Mash1 is primarily endogenously expressed in progenitors that become inhibitory neurons, yet iN cells generated via forced expression of Mash1 in combination with other factors include both excitatory subtype and dopamine neurons. It will be interesting to investigate the ability of other proneural genes to drive iN conversion and if a more appropriate choice of TFs will lead to a more extensive reprogramming. Given that Caiazzo et al. and our recent paper $[3,5]$ show that iN cells are flexible in terms of what neuronal subtype is generated, it appears likely that multiple kinds of neuronal subtypes can be generated via direct conversion if the appropriate TFs are identified. Control of neuronal subtype specification will in turn allow for possible treatment of a wider range of $\mathrm{CNS}$ disorders.

One advantage of direct conversion is that the iN cells do not divide once formed, meaning that the resulting cell population is likely to be a safer, nontumorgenic population of cells to graft. At the same time, lack of cell division can be a drawback since a relatively large starting population is required to obtain a workable number of neurons. It also means that integrating delivery systems, such as the lentiviral vectors used in the above mentioned studies, are poorly suited since the chance of a transforming integration event will be high. This has recently been solved in the iPS field by using non-stable genetic delivery, small molecules or direct delivery of mRNA [9]. As iN cells do not divide after reprogramming it is not clear if these approaches can be directly transferred or if the delivery techniques must be tailor-made. Still, it is probably safe to say that within a few years a suitable technical approach that is compatible with clinical use have been developed.

In summary, the work by Caiazzo et $a l$. is an additional report that points in the direction that induced neurons are a strong candidate to be a widely used tool in the future. Although much work remains before clinical translation is a reality, it certainly has opened up new avenues that will potentially allow patient-specific cell therapy. Exactly which reprogrammed cell type that will be best to use, being iPS, iN or a cell type yet to be identified remain to be decided, but it will certainly be interesting to follow their development into treatment strategies that appeared to be science fiction just a few years ago.

\section{Acknowledgments}

Our work is supported by grants from the
Swedish Research Council and European Community's 7th Framework Programme through Neurostemcell (nr. 22943).

\section{References}

1 Hargus G, Cooper O, Deleidi M, et al. Differentiated Parkinson patientderived induced pluripotent stem cells grow in the adult rodent brain and reduce motor asymmetry in Parkinsonian rats. Proc Natl Acad Sci USA 2010; 107:15921-15926.

2 Miura K, Okada Y, Aoi T, et al. Variation in the safety of induced pluripotent stem cell lines. Nat Biotechnol 2009; 27:743-745.

3 Caiazzo M, Dell'anno MT, Dvoretskova $\mathrm{E}$, et al. Direct generation of functional dopaminergic neurons from mouse and human fibroblasts. Nature 2011; 476:224-227.

4 Pang ZP, Yang N, Vierbuchen T, et al. Induction of human neuronal cells by defined transcription factors. Nature 2011; 476:220-223.

5 Pfisterer U, Kirkeby A, Torper O, et al. Direct conversion of human fibroblasts to dopaminergic neurons. Proc Natl Acad Sci USA 2011; 108:1034310348.

6 Vierbuchen T, Ostermeier A, Pang ZP, Kokubu Y, Südhof TC, Wernig M. Direct conversion of fibroblasts to functional neurons by defined factors. $\mathrm{Na}$ ture 2010; 463:1035-1041.

7 Kim K, Doi A, Wen B, et al. Epigenetic memory in induced pluripotent stem cells. Nature 2010; 467:285-290.

8 Parras CM, Schuurmans C, Scardigli R, Kim J, Anderson DJ, Guillemot F. Divergent functions of the proneural genes Mash1 and Ngn2 in the specification of neuronal subtype identity. Genes Dev 2002; 16:324-338.

9 Patel M, Yang S. Advances in reprogramming somatic cells to induced pluripotent stem cells. Stem Cell Rev 2010; 6:367-380. 